\title{
Sickle Cell Anemia Presenting with Vaso-Occlusive Pain: Should We Screen for COVID-19?
}

\author{
Mohammad Ali ${ }^{a}$ Lina Okar ${ }^{a} \quad$ Nabil E. Omar ${ }^{b} \quad$ Jabeed Parengal ${ }^{c}$ \\ Ashraf Soliman $^{d}$ Mohamed A. Yassin ${ }^{\mathrm{e}}$ \\ a Department of Medical Education, Hamad Medical Corporation, Doha, Qatar; ${ }^{b}$ Department of Pharmacy, National \\ Center for Cancer Care \& Research, Hamad Medical Corporation, Doha, Qatar; 'Department of Medicine, Infectious \\ Disease Section, Hamad Medical Corporation, Doha, Qatar; ${ }^{\mathrm{d} D e p a r t m e n t}$ of Pediatrics, Hamad Medical Corporation, \\ Doha, Qatar; ${ }^{\text {eDepartment }}$ of Medical Oncology, Hematology Section, National Center for Cancer Care \& Research, \\ Hamad Medical Corporation, Doha, Qatar
}

\section{Keywords}

Sickle cell disease - Vaso-occlusive - Coronavirus disease 19 . Acute chest syndrome

\begin{abstract}
Despite the widespread of coronavirus disease-19 (COVID-19) infection around the world, there are very scarce reported literature about the care of patients with a known diagnosis of hemoglobin disorders such as sickle cell disease (SCD) or thalassemia and confirmed COVID-19 infection. Thalassemia International Federation issued a position statement to include patients with thalassemia and SCD among the high-risk groups of patients. Here, we present an interesting case of a 42-year-old patient know to have SCD presenting with Vaso-occlusive (VOC) pain episode in the absence of COVID-19 signs and symptoms, who tested positive for COVID-19 infection and had a smooth recovery. This case highlights the importance of screening SCD patients presenting with VOC-related events even in the absence of COVID-19 signs and symptoms.

(c) 2021 The Author(s)

Published by S. Karger AG, Basel
\end{abstract}

\section{Introduction}

SARS-CoV-2 was first isolated in Wuhan, China. It has rapidly spread to other parts of the world and became a worldwide pandemic [1]. Coronavirus disease 19 (COVID-19) was declared as a global pandemic by the WHO on March 11, 2020 [2]. Patients with sickle cell disease (SCD) are among the high-risk group of patients according to a statement issued recently by Thalassemia International Federation (TIF). Categorizing those patients as high risk is based on many factors including the suppressed immunity due to compromised spleen function, the likelihood of COVID pneumonia/hypoxia to trigger acute chest syndrome (ACS) which is a major cause of morbidity and mortality in SCD patients, and the challenges of meeting the health care needs of this vulnerable group of patients at that time of a global crisis [3]. Here, we present an interesting case of a SCD patient presenting with VOC pain during the peak of COVID-19 spread in Qatar without remarkable respiratory or gastrointestinal symptoms.
C 2021 The Author(s).

Published by S. Karger AG, Basel

This article is licensed under the Creative Commons AttributionNonCommercial-NoDerivatives 4.0 International License (CC BYNC-ND) (http://www.karger.com/Services/OpenAccessLicense) Usage and distribution for commercial purposes as well as any distribution of modified material requires written permission.
Lina Okar

Family Medicine Department, Hamad Medical Corporation

PO Box 3050, Al Rayan Street

Doha (Qatar)

linaokar227@gmail.com 
Table 1. Investigations results

\section{Investigations \\ Chest X-ray}

Bilateral interstitial infiltrates and ground glass appearance with the impression of COVID-19 infection (Fig. 1)

ECG Normal with QTc 397 ms

\section{Blood tests}

Hemoglobin (13-17 g/dL)

RBCs $\left(4.5-5.5 \times 10^{6}\right)$

WBCs $\left(4-10 \times 10^{3} / \mathrm{UL}\right)$

PLT $\left(150-400 \times 10^{3} / \mathrm{UL}\right)$

7.6 after recovery 8.1

2.2

10.7

287

Lymphocytes count $\left(1-3 \times 10^{3} / \mathrm{UL}\right)$

Ferritin (8-252 mcg/L)

CRP (0-5 Umg/L)

D-dimer $(0.00-0.4 \mathrm{mcg} / \mathrm{L})$

LDH (135-214 U/L)

1.1

5.223 comparing with

1,349 2 months before

85

4.40

628

Renal function tests (urea/Cr)

$(2.1-8.8 \mathrm{mmol} / \mathrm{L})$

7.90

(44-80 Umoll/L)

64

\begin{tabular}{ll}
\hline ALT/AST & \\
$(0-33$ U/L) & 29 \\
$(0-32$ U/L) & 25 \\
\hline Albumin $(\mathbf{3 5 - 5 0} \mathbf{g} / \mathbf{L})$ & 33.9 \\
\hline G6PD & Normal \\
\hline
\end{tabular}

\section{Case Presentation}

A 42-year-old female with history of sickle cell anemia presented to the emergency department during COVID-19 pandemic with generalized body aches and left leg pain for 4 days with no associated symptoms of fever, cough, chest pain, or shortness of breath. She denied history of recent travel or contact with COVID-19 patients. She had 2 previous painful crises before presented as generalized body ache. Home medications included Hydroxyurea $400 \mathrm{mg}$ oral BID and folate $5 \mathrm{mg}$ oral daily. On presentation to the emergency department, her vitals were temperature 37 , HR $100 / \mathrm{min}$, oxygen saturation of arterial blood $99 \%$ on room air, and BP 160/100. Physical examination was unremarkable except for lower left leg tenderness. Initial laboratory investigations were sent. The patient was admitted as painful crisis and started on SC morphine as needed and IV fluids after that she described improvement in her pain.

Investigations results were as following: Table 1. Based on chest X-ray findings (Fig 1), it was decided to screen for COVID-19 infection. First COVID-19 nasopharyngeal swab was inconclusive, so it was repeated and tested positive. One day after admission, she started to complain of occasional dry cough, without fever, or shortness of breath. Following our local protocol for the treatment of COVID-19 infection, she was started on Azithromycin $500 \mathrm{mg}$ oral BID for 7 days, Hydroxychloroquine $400 \mathrm{mg}$ BID in the first day then once daily for the following 9 days, Ceftriaxone $2 \mathrm{~g} \mathrm{IV}$ for

Sickle Cell Anemia Presenting with

Vaso-Occlusive Pain

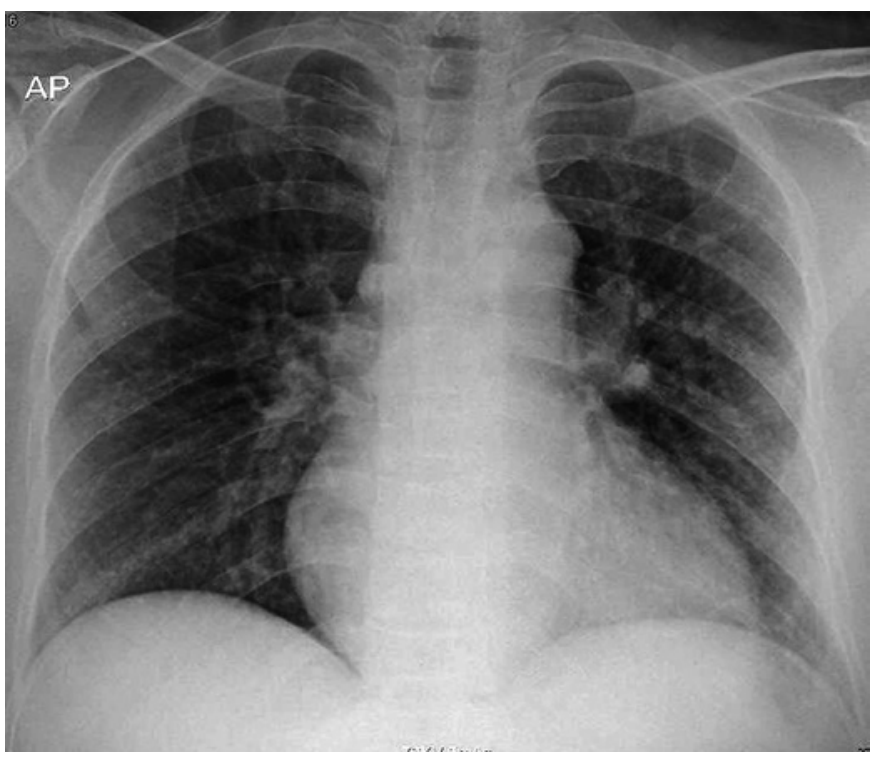

Fig. 1. Chest $\mathrm{X}$-ray showing $\mathrm{B} / \mathrm{L}$ interstitial infiltrates and ground glass appearance.

5 days and Oseltamivir 150 BID for 5 days, and enoxaparin $40 \mathrm{mg}$ SC daily.

During the 1-week hospital course, she had a smooth recovery without worsening of her symptoms and without needing oxygen supplementation or any escalation in management. Her pain subsided with IV hydration and analgesia after 2 days. One week later, she was discharged to a quarantine facility on Hydroxyurea $500 \mathrm{mg}$ daily and Folate $5 \mathrm{mg}$ daily.

\section{Discussion}

Coronaviruses are part of the Coronaviridae family that contains multiple viruses considered to be responsible for recent outbreaks, the last 1 caused by SARS-Cov2 presented as severe acute respiratory syndrome and the virus named COVID-19. It was first isolated in Wuhan in China due to increasing numbers of atypical pneumonia then, unfortunately, spread globally causing a pandemic [4].

$25-50 \%$ of COVID-19 cases may be asymptomatic or minimally symptomatic. Common symptoms are fever, cough, shortness of breath, headache, arthralgia, myalgia, sore throat, diarrhea, and loss of taste and smell. However, these symptoms might worsen over the second week. Few risk factors were suggested to be responsible for the poor COVID-19 infection prognosis as older age, comorbid chronic disease, and malignancy [5]. TIF issued a position statement to include patients with thalassemia and SCD among the high-risk groups of patients [3]. 
SCD is an inherited hemoglobin disorderthat correlates with high mortality and morbidity. The main pathology is mutation in the HBS gene which leads to deformity in RBCs. SCD prevalence was studied in the Gulf countries and Qatar and considered to have the highest prevalence ratio reaching 3.9\%. Regarding the genotype, two-thirds of diagnosed patients carry homozygous genotype, while others carry heterozygous. Thus, clinical presentation varies. Vaso-occlusive (VOC) crisis and cerebrovascular events increase mortality and morbidity in those patients along with other complications like renal failure, ACS, and severe anemia [6].

Episodes of acute pain due to VOC events are one of the most common reasons for emergency room visits in patients with SCD. Many patients have specific triggers for pain such as infection, cold, dehydration, stress, and menses. However, most of these painful episodes have no identifiable cause [7]. Viral infections are also among these triggers. H1N1 virus is considered to be a trigger to ACS during the pandemic in 2019. Depending on this experience and the outgrowing literature about COVID-19 infection, it is reasonable to think of COVID-19 as a trigger to VOC crisis also. The first case report highlighting this point was published by Frans et al. [9] in March $2020[8,9]$.

By reviewing the literature, we found that 19 SCD patients with COVID-19 were reported in the period between December 2019 and June 2020 none of them from the Gulf region. As our patient, 2 out of these 19 patients presented with typical VOC-related pain without fever, cough, shortness of breath, or other symptoms commonly encountered in patients with COVID-19 [10]. This observation highlights the importance of testing for COVID-19 infection in SCD patients presenting with only VOC events even if patients do not report history of contact with COVID-19 cases or exhibit symptoms of COVID-19 infection.

A UK experience of 10 patients concluded that SCD patients have favorable outcomes unless they suffer from another comorbidity. Patients with ACS received empirical antibiotics, RBCs exchange as well as blood transfusion, most of the cases with pneumonia required supplemental oxygen, in some cases Tocilizumab was used and showed a promising result in SCD patients with severe pneumonia $[9,10]$. In our patient, the clinical course was smooth and uncomplicated. Based on the available literature about SCD patients presenting with COVID-19 infection and VOC-related events, our patient received supportive care with hydration, analgesics, antibiotics, antivirals, and hydroxychloroquine based on our local protocol for the treatment of COVID-19 infection.

\section{Conclusion}

This case highlights the importance of testing at the time of a global pandemic for COVID-19 infection in patients with SCD presenting with VOC-related events even in the absence of COVID-19 signs and symptoms. We observed that early recognition and treatment of COVID-19 in this high-risk group of patients can contribute to a smooth recovery as seen in our patient. To the best of our knowledge, this is the first detailed case report of the coexistence of COVID-19 infection with SCD from the Middle East.

\section{Acknowledgements}

We would like to acknowledge the internal medicine residency program at Hamad Medical Corporation for the logistic support provided to publish this case report.

\section{Statement of Ethics}

Written informed consent was obtained from the patient for publication of this case report and any accompanying images. Case approved by HMC Medical Research Center.

\section{Conflict of Interest Statement}

The authors report no conflicts of interest.

\section{Funding Resources}

No funding was needed for this case. Open access will be paid by QNL.

\section{Authors Contribution}

M.A., L.O., N.O., and M.Y. were involved in data collection, analysis, and interpretation. M.A., L.O., and N.O. wrote the manuscript. M.Y. and J.P. critically revised the manuscript.

\footnotetext{
References $\quad 1$ Zheng J. SARS-CoV-2: an emerging coronavirus that causes a global threat. Int J Biol Sci. 2020 Mar 21;16(10):1678-85.

2 Cucinotta D, Vanelli M. WHO declares COVID-19 a pandemic. Acta Biome. 2020;91(1): $157-60$.
} 
3 Thalassemia International Federation. The COVID-19 pandemic and hemoglobin disorders: a contribution of thalassemia international federation to its global Patients' community (version III, updated). Thalassemia international federation. 2020.

4 Li H, Liu S-M, Yu X-H, Tang S-L, Tang C-K. Coronavirus disease 2019 (COVID-19): current status and future perspective. Int J Antimicrob Agents. 2020 Mar:55(5):105951.

5 Zumla A, Hui DS, Perlman S. Middle East respiratory syndrome. Lancet. 2015;386(9997): 995-1007.
6 Wali Y, Kini V, Yassin MA. Distribution of sickle cell disease and assessment of risk factors based on transcranial Doppler values in the Gulf region. Hematology. 2020;25(1):5562.

7 Soliman AT, Yasin M, El-Awwa A, Abdelrahman MO, De Sanctis V. Does blood transfusion affect pituitary gonadal axis and sperm parameters in young males with sickle cell disease? Indian J Endocrinol Metab. 2013; 17(6):962.
8 Sahu KK, Siddiqui AD, Cerny J. Managing sickle cell patients with COVID-19 infection: the need to pool our collective experience. $\mathrm{Br}$ J Haematol. 2020 Jul;190(2):e86-9.

9 Beerkens F, John M, Puliafito B, Corbett V, Edwards C, Tremblay D. COVID-19 pneumonia as a cause of acute chest syndrome in an adult sickle cell patient. Am J Hematol. 2020 Jul;95(7):E154-6.

10 McCloskey KA, Meenan J, Hall R, Tsitsikas DA. COVID-19 infection and sickle cell disease: a UK centre experience. Br J Haematol. $2020 \mathrm{Jul} ; 190(2):$ e57-5 